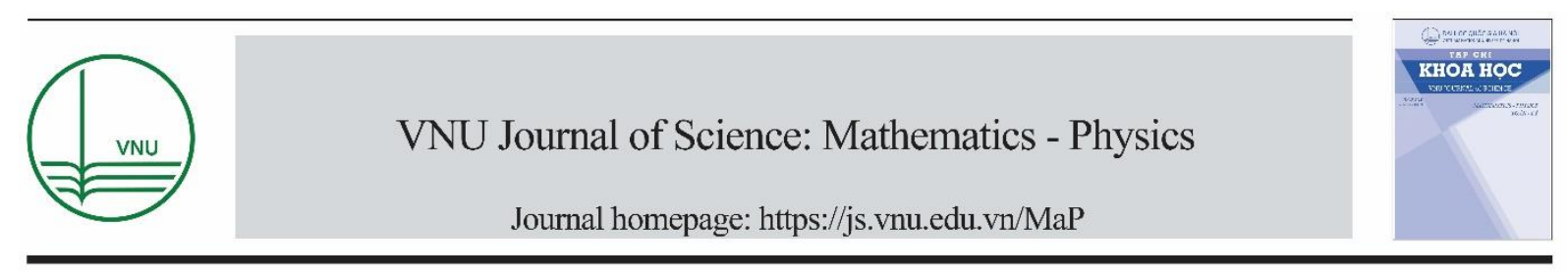

Original Article

\title{
Examination of the Fractal Model for Streaming Potential Coefficient in Porous Media
}

\author{
Luong Duy Thanh* \\ Thuy Loi University, 175 Tay Son, Dong Da, Hanoi, Vietnam \\ Received 26 September 2018 \\ Revised 26 October 2018; Accepted 17 December 2018
}

\begin{abstract}
In this work, the fractal model for the streaming potential coefficient in porous media recently published has been examined by calculating the zeta potential from the measured streaming potential coefficient. Obtained values of the zeta potential are then compared with experimental data. Additionally, the variation of the streaming potential coefficient with fluid electrical conductivity is predicted from the model. The results show that the model predictions are in good agreement with the experimental data available in literature. The comparison between the proposed model and the Helmholtz-Smoluchowski (HS) equation is also carried out. It is seen that the prediction from the proposed model is quite close to what is expected from the HS equation, in particularly at the high fluid conductivity or large grain diameters. Therefore, the model can be an alternative approach to obtain the zeta potential from the streaming potential measurements.
\end{abstract}

Keywords: Streaming potential, zeta potential, fractal, porous media.

\section{Introduction}

Streaming potential measurements play an important role in geophysical applications. For example, the streaming potential coefficient for various rock samples is one of the important factors in the evaluation of seismoelectric well logging [e.g., 1,2]. The streaming potential coefficient is also an important parameter in numerical simulations of seismoelectric exploration [e.g., 3, 4] and seismoelectric well logging [e.g., 5]. The streaming potential could be used to map subsurface flow and detect subsurface flow patterns in oil reservoirs [e.g., 6, 7], geothermal areas and volcanoes [e.g., 8, 9],

\footnotetext{
${ }^{*}$ Corresponding author.

E-mail address: luongduythanh2003@yahoo.com

https//doi.org/ 10.25073/2588-1124/vnumap.4306
} 
detection of contaminant plumes [e.g., 10, 11]. It has also been proposed to use the streaming potential monitoring to detect at distance the propagation of a waterfront in a reservoir [e.g., 12]. Monitoring of streaming potential anomalies has been proposed as a means of predicting earthquakes [e.g., 13, 14].

Fractal models on porous media have attracted increasing interests from many different disciplines [e.g., 15-22]. Recently, Luong et al. [23] have presented a fractal model for the streaming potential coefficient in porous media based on the fractal theory of porous media and on the streaming potential in a capillary. The proposed model has been applied to explain the dependence of the streaming potential coefficient on the grain size. The prediction is then compared with experimental data available in the literature and good agreement is found between them. However, the model is not yet examined more extensively.

In this work, the fractal model for the streaming potential coefficient in porous media presented in [23] is examined by calculating the zeta potential that is normally determined by a conventional Helmholtz- Smoluchowski (HS) equation. Obtained values are then compared with experimental data available in literature. The result shows that the predicted zeta potential is in good agreement with the experimental data. The comparison between the proposed model and the HS equation is also carried out by plotting the ratio of the SPC as a function of particle diameter. It is shown that that the proposed model is able to reproduce the similar result to the HS equation, in particular at the high fluid conductivity or large grain diameters.

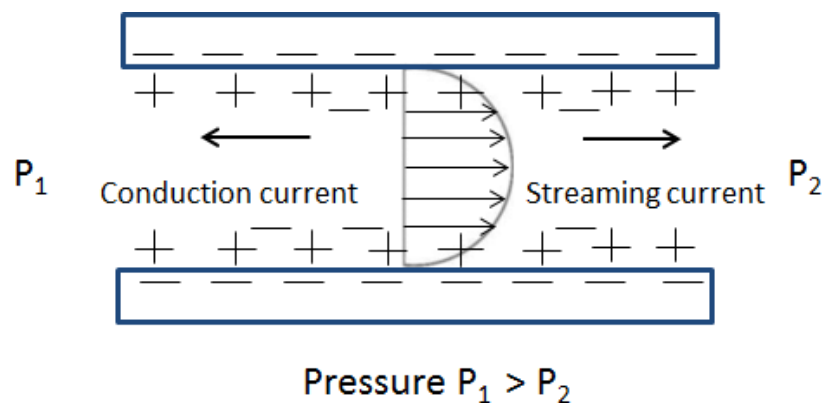

Figure 1. Development of streaming potential when an electrolyte is pumped through a capillary (a porous medium is made of an array of capillaries).

\section{Theoretical background}

When a porous medium is saturated with an electrolyte, an electric double layer is formed on the interface between the solid and the fluid. Some ions are absorbed into the solid surface and other ions remain movable in the fluid. When a pressure difference is applied across a fluid-saturated porous medium, the relative motion happens between the pore fluid and solid grain surface. Then the net ions of the diffuse layers move along with the flowing fluid at the same time. This movement of the net ions generates a convection current (called streaming current) in the capillaries (a porous medium can be approximated as an array of capillaries). The movement of the ions in the diffuse layer also makes the separation of the positive and negative ions. Thus, an electric potential (streaming potential) is created and that induces a conduction current in the opposite direction to the streaming current as shown in Fig. 1). The streaming potential coefficient (SPC) is a key parameter that relates the pressure 
difference $(\Delta P)$ and the streaming potential difference $(\Delta V)$ when the total current density $(j)$ is zero as [24]

$$
C_{S}=\left.\frac{\Delta V}{\Delta P}\right|_{j=0}
$$

The streaming potential coefficient in porous media is given by [e.g., 25, 26]

$$
C_{S}=\frac{\varepsilon_{r} \varepsilon_{o} \zeta}{\eta \sigma_{e f f}}
$$

where $\varepsilon_{r}$ is the relative permittivity of the fluid, $\varepsilon_{o}$ is the dielectric permittivity in a vacuum, $\eta$ is the dynamic viscosity of the fluid, $\sigma_{\text {eff }}$ is the effective conductivity, and $\zeta$ is the zeta potential which is the electrical potential associated with the counter charge in the electrical double layer at the mineralfluid interface. The effective conductivity including the bulk fluid conductivity and the surface conductivity is given by [e.g., 25, 27, 28 ]

$$
\sigma_{e f f}=K_{b}+\frac{2 K_{s}}{\Lambda}
$$

where $K_{b}$ is the bulk fluid conductivity, $K_{s}$ the specific surface conductance, $\Lambda$ is a characteristic length scale that describes the size of the pore network. There have been several models that relate the characteristic length scale to grain diameter. One is given by [29]

$$
\Lambda=\frac{d}{2 m(F-1)},
$$

where $d$ is the mean grain diameter, $F$ is the formation factor (no units), $m$ is the cementation exponent of porous media (no units).

$$
C_{S}=\frac{\varepsilon_{r} \varepsilon_{o} \zeta}{\eta\left(K_{b}+\frac{2 K_{s}}{\Lambda}\right)}=\frac{\varepsilon_{r} \varepsilon_{o} \zeta}{\eta\left(K_{b}+\frac{2 m K_{s}(F-1)}{d}\right)} .
$$

Consequently, Eq. (2) can be rewritten as

Eq. (2) and therefore, Eq. (5) are known as the modified HS equation as mentioned above.

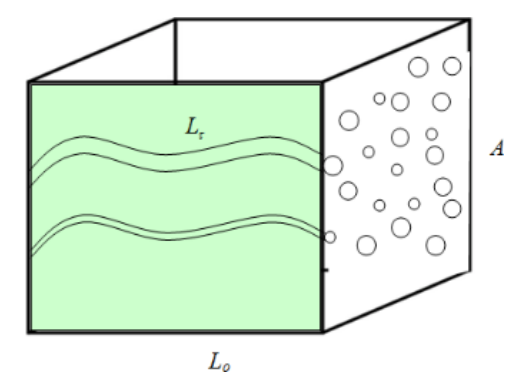

Figure 2. A porous medium composed of a large number of tortuous capillaries with random radius. 


\section{Fractal theory for porous media}

It has been shown that many natural porous media usually have extremely complicated and disordered pore structure with pore sizes extending over several orders of magnitude and their pore spaces have the statistical self-similarity and fractal characters [e.g., 15, 18]. Fractal models provide an alternative and useful means for studying the transport phenomenon and analyzing the macroscopic transport properties of porous media. To derive the streaming potential coefficient in porous media, a representative elementary volume (REV) of a cylinder of radius $r_{r e v}$ and length $L_{r e v}$ is considered [30]. The pores are assumed to be circular capillary tubes with radii varying from a minimum pore radius $r_{\min }$ to a maximum pore radius $r_{\max }\left(0<r_{\min }<r_{\max }<r_{\text {rev }}\right)$. A porous medium is assumed to be made up of an array of tortuous capillaries with different sizes (see Fig. 2). The cumulative size distribution of pores is assumed to obey the following fractal law $[18,21,22,30]$ :

$$
N(\geq r)=\left(\frac{r_{\text {max }}}{r}\right)^{D_{f}}
$$

where $N$ is the number of capillaries (whose radius $\geq r$ ) in a fractal porous media, $D_{f}$ is the fractal di- mension for pore space $\left(0<D_{f}<2\right.$ in two-dimensional space and $0<D_{f}<3$ in three dimensional space $[18,21,22])$. Eq. (6) implies the property of self-similarity of porous media, which means that the value of $D_{f}$ from Eq. (6) remains constant across a range of length scales. As there are numerous capillaries in porous media, Eq. (6) can be considered as a continuous function of the radius.

Differentiating Eq. (6) with respect to $r$ yields

$$
-d N=D r_{\max }^{D_{f}} r^{-D_{f}-1} d r
$$

where $-d N$ represents the number of pores from the radius $r$ to the radius $r+d r$. The minus (-) in Eq. (7) implied that the number of pores decreases with the increase of pore size.

The fractal dimension for pore space is expressed as [e.g., 18, 21, 22]

$$
D_{f}=2-\frac{\ln \phi}{\ln \alpha}
$$

where $\phi$ is the porosity of porous media and $\alpha$ is the ratio of the minimum pore radius to the maximum pore radius $\left(\alpha=r_{\min } / r_{\max }\right)$. For most porous media, it is stated that $\alpha \approx 10^{-2}$ or $<10^{-2}$ [e.g., $18,21,22]$.

Cai et al. [19] proposed an expression to calculate maximum radius as

$$
r_{\max }=\frac{d}{8}\left[\sqrt{\frac{2 \phi}{1-\phi}}+\sqrt{\frac{\phi}{1-\phi}}+\sqrt{\frac{\pi}{4(1-\phi)}-1}\right],
$$

where $d$ is the mean grain diameter in porous media.

\section{Streaming current in porous media}

The streaming current in a capillary of radius $r$ under a fluid pressure difference $\left(\Delta P_{\text {rev }}\right)$ across the REV is given by [31, 32]

$$
i_{s}(r)=\frac{\pi r^{2} \cdot \varepsilon \varepsilon_{o} \zeta}{\eta} \cdot \frac{\Delta P_{r e v}}{L_{\tau}}\left[1-\frac{2 \lambda I_{1}(r / \lambda)}{r I_{0}(r / \lambda)}\right]
$$


where $\Delta P$ is the pressure difference across the capillary; $L_{\tau}$ is the real length of the tortuous capillaries; $I_{0}$ and $I_{1}$ are the zero-order and the first-order modified Bessel functions of the first kind, respectively and $\lambda$ is the Debye length that depends solely on the properties of the fluid and not on the properties of the solid surface [e.g., 33, 34].

For electrolytes with concentrations in the range of $1 \mathrm{mM}$ to $0.1 \mathrm{M}$ (typical concentrations for aqueous solutions saturating rocks or soils), the Debye length varies between $10 \mathrm{~nm}$ and $1 \mathrm{~nm}$ at $25^{\circ} \mathrm{C}$ [e.g., 34]. In general, the pore radius of rocks is around tens of micrometer [e.g., 35]. The Debye length is typically much smaller than pore sizes of a majority of rocks and soils. In this case, $I_{1}(r / \lambda) / I_{0}(r / \lambda)$ can be neglected. Under that condition, Eq. (10) is simplified as

$$
i_{s}(r)=\frac{\pi r^{2} . \varepsilon \varepsilon_{o} \zeta}{\eta} \cdot \frac{\Delta P_{r e v}}{L_{\tau}}=\frac{\pi r^{2} \cdot \varepsilon \varepsilon_{o} \zeta}{\eta} \cdot \frac{\Delta P_{r e v}}{L_{r e v} \cdot \tau},
$$

where $L_{\tau}$ is related to the length of the representative elementary volume $L_{r e v}$ as $L_{\tau}=\tau L_{r e v}$ [e.g., 36] ( $\tau$ is the tortuosity of the capillary).

The streaming current through the representative elementary volume of the porous medium is the sum of the streaming currents over all individual capillaries and is given by

$$
I_{s}=\int_{r_{\min }}^{r_{\max }} i_{s}(r)(-d N) \text {. }
$$

Substituting Eq. (7) and Eq. (11) into Eq. (12), the following is obtained

$$
I_{s}=\int_{r_{\min }}^{r_{\max }} \frac{\pi \cdot \varepsilon \varepsilon_{o} \zeta}{\eta} \cdot \frac{\Delta P_{r e v}}{\tau \cdot L_{r e v}} D_{f} r_{\max }^{D_{f}} r^{1-D_{f}} d r=\frac{\pi \cdot \varepsilon \varepsilon_{o} \zeta}{\eta} \cdot \frac{\Delta P_{r e v}}{\tau \cdot L_{r e v}} \cdot \frac{D_{f}}{2-D_{f}} r_{\max }^{2}\left(1-\alpha^{2-D_{f}}\right) .
$$

\subsection{Conduction current in porous media}

The streaming current is responsible for the streaming potential. As a consequence of the streaming current, a potential difference called streaming potential $(\Delta V)$ will be set up between the ends of the capillary. This streaming potential in turn will cause an electric conduction current opposite in direction with the streaming current (see Fig. 1). The conduction current when taking into account both bulk conduction and surface conduction of the capillary is given by [37, 38]

$$
i_{c}(r)=\frac{\pi \Delta V}{L_{r e v} \tau}\left[K_{b} r^{2}+2 K_{s} r\right]
$$

The conduction current through the representative elementary volume is given by

$$
I_{c}=\int_{r_{\min }}^{r_{\max }} i_{c}(r)(-d N)=\frac{D_{f} \pi \Delta V}{L_{r e v} \tau}\left[\frac{K_{b}}{2-D_{f}} r_{\max }^{2}\left(1-\alpha^{2-D_{f}}\right)+\frac{2 K_{s}}{1-D_{f}} r_{\max }\left(1-\alpha^{1-D_{f}}\right)\right]
$$

\subsection{Streaming potential coefficient in porous media}

At steady state, the following is obtained

$$
I_{\mathrm{s}}=\mathrm{I}_{\mathrm{c}}
$$

Combining Eq. (12), Eq. (15) and Eq. (16) yields 


$$
\Delta V=\frac{\varepsilon_{0} \zeta \Delta P}{\eta\left[K_{b}+\frac{2 K_{s}}{r_{\max }} \cdot \frac{2-D_{f}}{1-D_{f}} \cdot \frac{1-\alpha^{1-D_{f}}}{1-\alpha^{2-D_{f}}}\right]} .
$$

The streaming potential coefficient in the fractal model is obtained as

$$
C_{S}=\frac{\Delta V}{\Delta P}=\frac{\varepsilon \varepsilon_{o} \zeta}{\eta\left[K_{b}+\frac{2 K_{s}}{r_{\max }} \cdot \frac{2-D_{f}}{1-D_{f}} \cdot \frac{1-\alpha^{1-D_{f}}}{1-\alpha^{2-D_{f}}}\right]}
$$

Eq. (18) is the fractal model for the SPC already presented in [23].

Table 1. The parameters of sandstone samples reported in [39].

\begin{tabular}{llll}
\hline Sample ID & Porosity (percent) & Formation factor (-) & Permeability $(\mathrm{mD})$ \\
\hline D1 & 30.6 & 9.131 & 1028 \\
D2 & 30.2 & 7.873 & 1435 \\
D3 & 30.9 & 8.415 & 1307 \\
D4 & 32.1 & 8.644 & 1152 \\
D5 & 29.8 & 8.319 & 456 \\
D6 & 31.0 & 8.497 & 978 \\
D7 & 29.4 & 8.156 & 594 \\
D8 & 31.0 & 11.792 & 2785 \\
D9 & 29.3 & 9.308 & 1491 \\
D10 & 31.5 & 8.793 & 3241 \\
\hline
\end{tabular}

\section{Discussion}

To examine the fractal model for the SPC, experimental data reported in [39] for ten cylindrical sandstone samples $(25 \mathrm{~mm}$ in diameter and around $20 \mathrm{~mm}$ in length) saturated by six different salinities $(0.02,0.05,0.1,0.2,0.4$ and $0.6 \mathrm{~mol} / \mathrm{NaCl}$ solutions $)$ are used. Parameters of the sandstone samples are reported in [39] and re-shown in Table 1. The measured SPC at the different salinities presented in [39] is also re-shown in Table 2.

Table 2. The magnitude of the SPC (in $\mathrm{nV} / \mathrm{Pa})$ at different electrolyte concentrations $\left(C_{f}\right.$ in mol/l) reported in [39].

\begin{tabular}{lllllll}
\hline Sample ID & 0.02 & 0.05 & 0.1 & 0.2 & 0.4 & 0.6 \\
\hline D1 & 54.01 & 20.40 & 14.73 & 10.05 & 7.02 & 3.11 \\
D2 & 83.74 & 23.18 & 18.91 & 11.03 & 8.35 & 3.47 \\
D3 & 80.85 & 22.05 & 16.31 & 10.05 & 6.27 & 3.26 \\
D4 & 48.69 & 21.71 & 18.85 & 12.05 & 8.15 & 3.81 \\
D5 & 39.87 & 19.33 & 13.95 & 8.95 & 7.75 & 3.61 \\
D6 & 59.24 & 22.54 & 15.32 & 10.26 & 7.91 & 3.62 \\
D7 & 40.33 & 16.51 & 12.35 & 9.76 & 7.65 & 4.41 \\
D8 & 212.92 & 42.46 & 27.50 & 18.48 & 10.87 & 4.26 \\
D9 & 97.59 & 35.77 & 22.15 & 10.51 & 6.92 & 3.59 \\
D10 & 224.81 & 57.88 & 30.03 & 15.66 & 9.61 & 3.76 \\
\hline
\end{tabular}


To obtain the zeta potential from the model - Eq. (18), one needs to know the SPC (see Table 2), the electrical conductivity, the surface conductivity and the fractal parameters of the porous rocks $(\alpha$, $D_{f}$ and $r_{\max }$ ). In the model, $\varepsilon_{r}$ is taken as 80 (no units) [40]; $\varepsilon_{o}$ is taken as $8.854 \times 10^{-12} \mathrm{~F} / \mathrm{m} \mathrm{[40];} \eta$ is taken as 0.001 Pa.s [40]; $\alpha$ is taken as 0.00001 (no units) because of the best fit to the experimental data (this value is also comparable to that used in [21] for rocks of Fontainebleau sandstone). Electrical conductivity of the $\mathrm{NaCl}$ solutions (the original stock solutions) is not mentioned in [39] but it can be obtained from the electrolyte concentration using $K_{b}=10 \mathrm{C}_{f}$ (that is valid in the range $10^{-6} \mathrm{M}$ $<\mathrm{C}_{f}<1 \mathrm{M}$ and $15^{\circ} \mathrm{C}<$ temperature $<25^{\circ} \mathrm{C}$ ) [41]. However, when the stock solutions are passed through the rock samples and become equilibrated with it. Geochemical interactions occur between solid grains and the pore fluid that are associated with dissolution and precipitation.

These change the salinity, composition, and $\mathrm{pH}$ of the pore fluid $[42,43]$. It is found that there is a significant increase of around $30 \%$ in the salinity of low salinity stock solutions $\left(\mathbf{C}_{f}<0.2 \mathrm{~mol} / \mathrm{l}\right)$ after equilibration with silica-based rocks [42]. While a reduction in pore fluid salinity could also occur at high salinity stock solutions $\left(\mathbf{C}_{f}>0.2 \mathrm{~mol} / \mathrm{l}\right)$ due to precipitation. Therefore, the actual electrical conductivity for $C_{f}<0.2 \mathrm{~mol} / 1(0.02 \mathrm{~mol} / 1,0.05 \mathrm{~mol} / \mathrm{l}$ and $0.1 \mathrm{~mol} / \mathrm{l})$ is approximately obtained by the relation $K_{b}=10 \mathrm{C}_{f} / 0.7$; for $\mathrm{C}_{f}=0.2 \mathrm{~mol} / \mathrm{l}$ by $K_{b}=10 \mathrm{C}_{f}$ and for $\mathrm{C}_{f}>0.2 \mathrm{~mol} / \mathrm{l}(0.4 \mathrm{~mol} / \mathrm{l}$ and 0.6 $\mathrm{mol} / \mathrm{l})$ by $K_{b}=10 \mathrm{C}_{f} / 1.3$ [42]. The specific surface conductance almost does not vary with salinity at salinity higher than $10^{-3} \mathrm{~mol} / \mathrm{l}$ [44]. Therefore, the surface conductance is assumed to be constant over the range of electrolyte concentration used in this work and taken as $8.9 \times 10^{-9} \mathrm{~S}$ for the silica-based samples [44]. This value is comparable to those reported in literature (e.g., $K_{s}=4.0 \times 10^{-9} \mathrm{~S}$ [27] or $5 \times 10^{-9} \mathrm{~S}$ [45]). The fractal dimension $D_{f}$ is determined from Eq. (8) with porosity reported in Table 1. The maximum radius $r_{\max }$ is determined from Eq. (9) in which the mean diameter of particles in porous media is calculated from Eq. (4)

$$
d=2 m(F-1) \Lambda
$$

where $m$ is taken as 1.9 for consolidated sandstones [46] and the $\Lambda$ is linked to the permeability of the porous medium $\left(k_{o}\right)$ as follows [47]

$$
\Lambda=\sqrt{8 F k_{o}}
$$

Table 3. The magnitude of the zeta potential (in mV) obtained from Eq. (18) at different electrolyte concentrations $\left(C_{\mathrm{f}}\right.$ in $\left.\mathrm{mol} / \mathrm{l}\right)$.

\begin{tabular}{lllllll}
\hline Sample ID & 0.02 & 0.05 & 0.1 & 0.2 & 0.4 & 0.6 \\
\hline D1 & 76.97 & 40.85 & 43.66 & 42.68 & 39.72 & 24.77 \\
D2 & 126.04 & 48.27 & 57.57 & 47.73 & 47.91 & 27.92 \\
D3 & 116.41 & 44.47 & 48.59 & 42.83 & 35.56 & 26.02 \\
D4 & 71.28 & 44.31 & 56.61 & 51.64 & 46.43 & 30.50 \\
D5 & 87.63 & 53.64 & 52.13 & 44.92 & 49.84 & 31.55 \\
D6 & 93.93 & 48.75 & 47.87 & 45.22 & 46.02 & 29.42 \\
D7 & 81.59 & 42.93 & 43.99 & 47.28 & 47.86 & 37.77 \\
D8 & 171.28 & 58.66 & 64.45 & 67.01 & 54.75 & 31.29 \\
D9 & 118.89 & 64.22 & 61.08 & 42.46 & 37.72 & 27.85 \\
D10 & 226.96 & 91.84 & 76.54 & 60.00 & 50.38 & 28.39 \\
\hline
\end{tabular}

Eq. (19) is now rewritten as 


$$
d=2 m(F-1) \sqrt{8 F k_{o}} .
$$

Therefore, the mean diameter of particles in porous media is determined with the knowledge of the cementation exponent $m$, the formation factor $F$ and permeability $k_{o}$ (see Table 1 ).

(a)

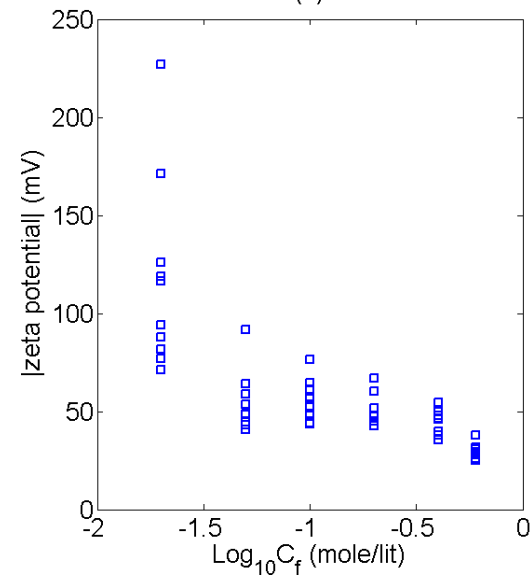

(b)

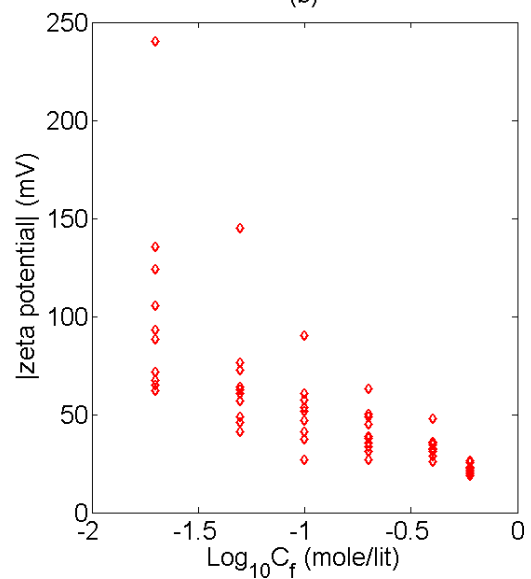

Figure 3. The zeta potential at different electrolyte concentrations for all samples:

(a) is obtained from the fractal model and (b) is obtained from Table 4 in [39].

Table 3 shows the magnitude of the zeta potential obtained from the fractal model at different electrolyte concentrations. The comparison between the zeta potential predicted from the model (Table 3) and experimental data reported in [39] is shown in Fig. 3. It is seen that the the model can reproduce the main trend of experimental data reported in [39] (Table 4 in their paper). For more details, the variation of zeta potential with electrolyte concentration predicted from the model and from [39] is shown in Fig. 4 for the representative sample D9. By fitting experimental data, the relation between the zeta potential and the electrolyte concentration is found to be $\zeta=-10+55 \log 10\left(C_{f}\right)$ for the sample D9 ( $\zeta$ is in $\mathrm{mV}$ and $C_{f}$ is in mol/l). Fig. 5 shows the variation of the SPC with the fluid electrical conductivity for the sample D9 in which the symbols are from [39] and the solid line is predicted from the model. It is seen that the model can quantitatively explain the experimental data well.

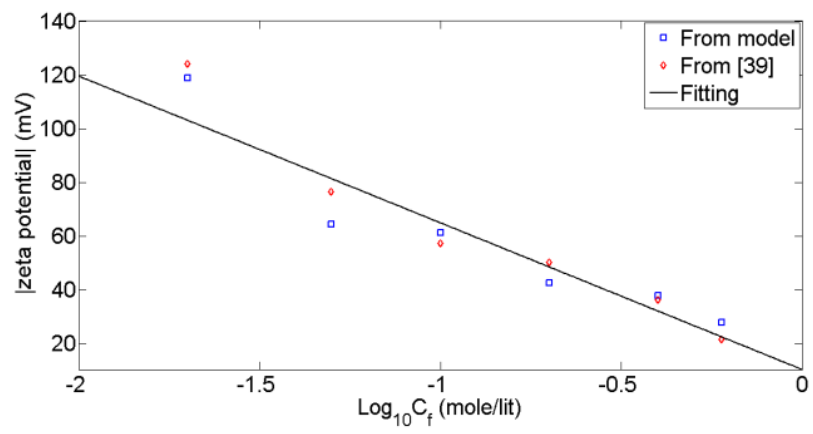

Figure 4. The variation of the zeta potential with the fluid electrical conductivity for the representative sample D9 deduced from the model and from [39]. 


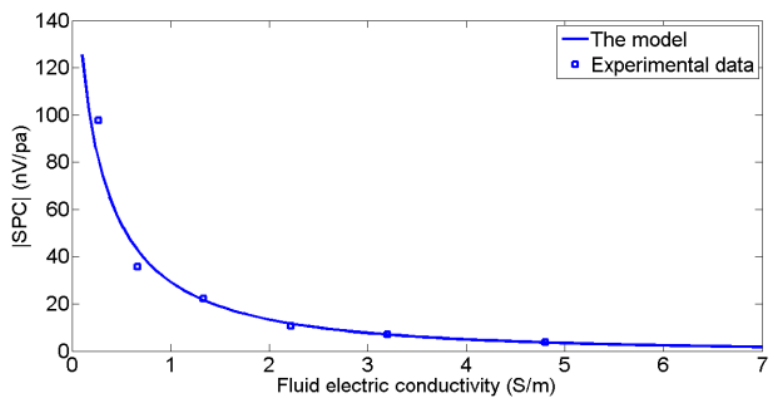

Figure 5. The variation of the SPC with the fluid electrical conductivity for the representative sample D9 deduced from the model and from [39].

Additionally, Fig. 6 shows the dependence of the SPC on the fluid electrical conductivity for three glass bead packs with different particle diameters $(d=56 \mu \mathrm{m}$ denoted by S1a, $d=72 \mu \mathrm{m}$ denoted by S1b and $d=512 \mu \mathrm{m}$ denoted by S5) obtained from [48] (see the symbols) and the model (the solid lines). In the model, $\varphi=0.4$ [48]; $K_{s}=4.0 \times 10^{-9} \mathrm{~S}$ [48]; the relation between the zeta potential and the fluid electrical conductivity $\zeta=14.6+29.1 \times \log _{10}\left(K_{b}\right)$ [48]; and $\alpha=0.01$ for unconsolidated porous samples such as sand packs [e.g., 19, 21,22] are used. The fractal dimension $\boldsymbol{D}_{f}$ is determined via Eq. (8). The maximum radius $r_{\max }$ is determined from Eq. (9) with the knowledge of particle diameter $d$ and porosity $\varphi$. The result shows that the model is able to reproduce the main trend as the experimental data.

The ratio of the SPC presented in Eq. (5) and that presented in Eq. (18) is obtained as below

$$
R=\frac{. K_{b}+\frac{2 K_{s}}{r_{\max }} \cdot \frac{2-D_{f}}{1-D_{f}} \cdot \frac{1-\alpha^{1-D_{f}}}{1-\alpha^{2-D_{f}}}}{K_{b}+\frac{4 m K_{s}(F-1)}{d}}
$$

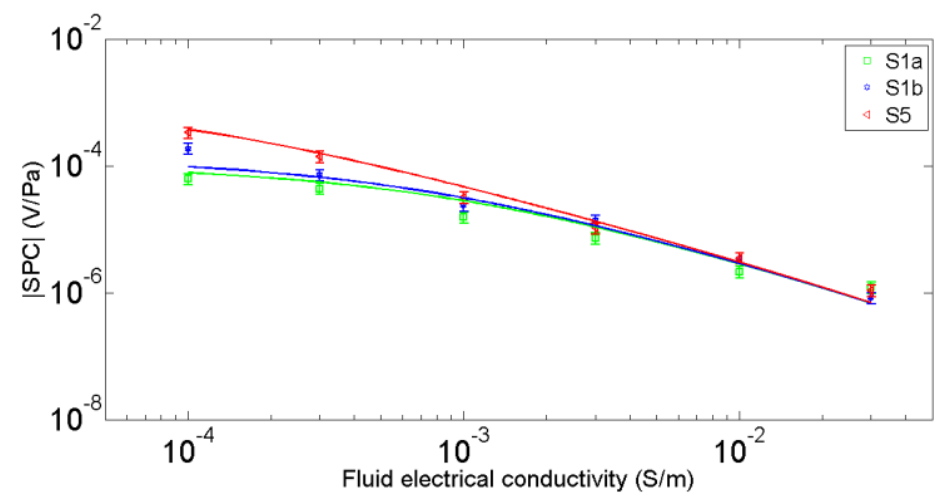

Figure 6. The variation of the SPC with the fluid electrical conductivity for three different sand packs obtained from [48] (symbols) and from the model (solid lines).

To predict the variation of $R$ with particle diameter for unconsolidated porous samples, $\phi$ is taken as $0.4, \alpha$ is taken as $0.01, K_{s}$ is taken as $4 \times 10^{-3} \mathrm{~S}$ for silica particle and $m$ is taken as 1.5 [49]. Fig. 7 shows the ratio of the SPC as a function of diameter $d$ at three different electrical conductivities $\left(K_{b}=\right.$ 
$2.0 \times 10^{-3} \mathrm{~S} / \mathrm{m}, 2.0 \times 10^{-2} \mathrm{~S} / \mathrm{m}$ and $2.0 \times 10^{-1} \mathrm{~S} / \mathrm{m}$ ). The result shows that the prediction from the proposed model is quite close to what is predicted from the HS equation, in particularly at the high fluid conductivity or large grain diameters. The reason is that the surface conductivity can be negligible for those cases.

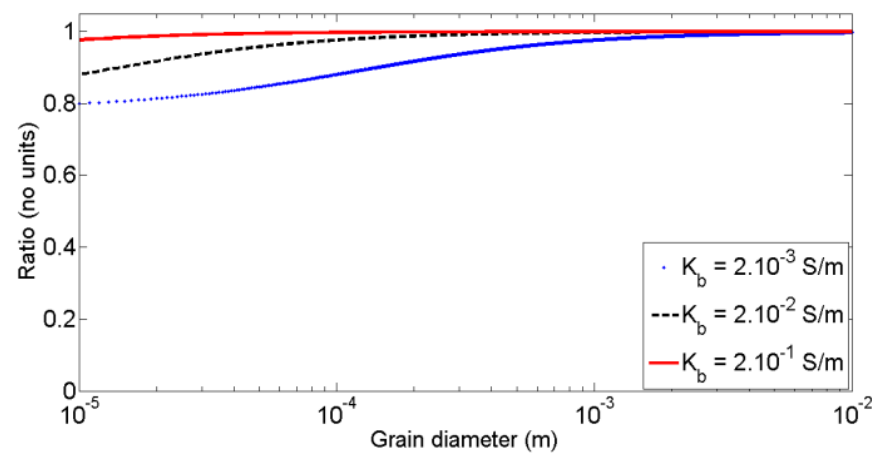

Figure 7. The ratio of the streaming potential coefficient as a function of particle diameter at three different electrical conductivities.

\section{Conclusions}

We examine the fractal model for the streaming potential coefficient in porous media recently published by deducing the zeta potential from the SPC. Obtained values of the zeta potential are then compared with measured data for ten rock samples saturated by six different salinities. Additionally, the variation of the SPC with fluid electrical conductivity is predicted from the model and compared with experimental data. The results show that the model predictions are in good agreement with the experimental data available in literature. The comparison between the proposed model and the HS equation is also carried out by plotting the ratio of the SPC as a function of particle diameter. It is seen that that the prediction from the proposed model is quite close to what is predicted from the HS equation, in particularly at the high fluid conductivity or large grain diameters. It is suggested that the model can be an alternative approach to obtain the zeta potential without empirical constants (the formation factor $F$ and the cementation exponent $m$ ) besides the conventional HS equation.

\section{Acknowledgments}

This research is funded by Vietnam National Foundation for Science and Technology Development (NAFOSTED) under grant number 103.99-2016.29

\section{References}

[1] Z. Zhu, M.W. Haartsen, M.N. Toksz, Experimental studies of electrokinetic conversions in fluid - saturated borehole models, Geophysics 64 (1999) 1349-1356.

[2] Z. Zhu, M.N. Toksz, D.R. Burns, Electroseismic and seismoelectric measurements of rock samples in a water tank, Geophysics 73 (2008) E153-E164. 
[3] M. Haartsen, S.R. Pride, Electroseismic waves from point sources in layered media, Journal of Geophysical Research 102 (1997) 24745-24769.

[4] S. Garambois, M. Dietrich, Full waveform numerical simulations of seismoelectromagnetic wave conversions in fluid-saturated stratified porous media, Journal of Geophysical Research: Solid Earth 107 (2002).

[5] W. Guan, H. Hu, Finite-difference modeling of the electroseismic logging in a fluid-saturated porous formation, Journal of Computational Physics 227 (2008) 5633-5648.

[6] B. Wurmstich, F.D. Morgan, Modeling of streaming potential responses caused by oil well pumping, Geophysics 59 (1994) 46-56.

[7] K. Titov, A. Revil, P. Konosavsky, S. Straface, S. Troisi, Numerical modelling of self-potential signals associated with a pumping test experiment, Geophysical Journal International 162 (2005) 641-650.

[8] R.F. Corwin, D.B. Hoover, The self-potential method in geothermal exploration, Geophysics 44 (1979) $226-245$.

[9] G. Mauri, G. Williams-Jones, G. Saracco, Depth determinations of shallow hydrothermal systems by selfpotential and multi-scale wavelet tomography, Journal of Volcanology and Geothermal Research 191 (2010) 233-244.

[10] P. Martinez-Pagan, A. Jardani, A. Revil, A. Haas, Self-potential monitoring of a salt plume, geophysics 75 (2010) WA17-WA25.

[11] V. Naudet, A. Revil, J.Y. Bottero, P. Bgassat, Relationship between self-potential (SP) signals and redox conditions in contaminated groundwater, Geophysical Research Letters 30 (2003).

[12] J.H. Saunders, M.D. Jackson, C.C. Pain, Fluid flow monitoring in oil fields using downhole measurements of electrokinetic potential, Geophysics 73(5) (2008) E165-E180.

[13] H. Mizutani, T. Ishido, T. Yokokura, S. Ohnishi, Electrokinetic phenomena associated with earthquakes, Geophys. Res. Lett. 3 (1976).

[14] M. Trique, P. Richon, F. Perrier, J. Avouac, J.C.Sabroux, Radon emanation and electric potential variations associated with transient deformation near reservoir lakes, Nature (1999) 137-141.

[15] B.B. Mandelbrot, The Fractal Geometry of Nature, W.H. Freeman, New York, 1982.

[16] J. Feder, A. Aharony, Fractals in Physics, North Holland, Amsterdam, 1989.

[17] P. Xu, A discussion on fractal models for transport physics of porous media, Fractals 23 (2015).

[18] B. Yu, P. Cheng, A fractal permeability model for bi-dispersed porous media, International Journal of Heat and Mass Transfer 45 (2002) 2983-2993.

[19] J.C. Cai, X.Y. Hu, D.C. Standnes, L.J. You, An analytical model for spontaneous imbibition in fractal porous media including gravity, Colloids and Surfaces, A: Physicocemical and Engineering Aspects 414 (2012) 228-233.

[20] J.C. Cai, L.J. You, X.Y. Hu, J. Wang, R.H. Peng, Prediction of effective permeability in porous media based on spontaneous imbibition effect, International Journal of Modern Physics C 23 (2012).

[21] M. Liang, S. Yang, B. Yu, A fractal streaming current model for charged microscale porous media, Journal of Electrostatics 72 (2014).

[22] M. Liang, S. Yang, T. Miao, B. Yu, Analysis of electroosmotic characters in fractal porous media, Chemical Engineering Science 127 (2015).

[23] L.D. Thanh, P.V. Do, N.V. Nghia, N.X. Ca, A fractal model for streaming potential coefficient in porous mediaGeophysical Prospecting 66 (2018) 753-766.

[24] W.R. Sill, Self-potential modeling from primary flows, Geophysics 48 (1983) 76-86.

[25] F.D. Morgan, E.R. Williams, T. R. Madden, Streaming potential properties of westerly granite with applications, Journal of Geophysical Research 94 (1989) 12.449-12.461.

[26] L. Jouniaux, T. Ishido, Electrokinetics in Earth Sciences: A Tutorial, International Journal of Geophysics, Article ID 286107 (2012) 16 pages, doi:10.1155/2012/286107.

[27] P.W.J. Glover, N. Dery, Streaming potential coupling coefficient of quartz glass bead packs: Dependence on grain diameter, pore size, and pore throat radius, Geophysics 75 (2010) F225-F241.

[28] T. Ishido, H. Mizutani, Experimental and Theoretical Basis of Electrokinetic Phenomena in Rock-Water Systems and Its Applications to Geophysics, Journal of Geophysical Research 86 (1981) 1763-1775.

[29] A. Revil, L.M. Cathles III, P.D. Manhardt, Permeability of shaly sands, Water Resources Research 3 (1999) 651662. 
[30] L. Guarracino, D. Jougnot, A Physically Based Analytical Model to Describe Effective Excess Charge for Streaming Potential Generation in Water Saturated Porous Media, Journal of Geophysical Research: Solid Earth 123 (2018) 52-65.

[31] C. Rice, R. Whitehead, Electrokinetic flow in a narrow cylindrical capillary, J. Phys. Chem. 69 (1965) 4017 4024.

[32] T. Paillat, E. Moreau, P.O.Grimaud, G. Touchard, Electrokinetic phenomena in porous media applied to soil decontamination, IEEE Transactions on Dielectrics and Electrical Insulation 7 (2000) 693-704.

[33] R.J. Hunter, Zeta Potential in Colloid Science, Academic, New York, 1981.

[34] J. Israelachvili, Intermolecular and Surface Forces, Academic Press, 1992.

[35] X. Hu, S. Hu, F. Jin, S. Huang, Physics of Petroleum Reservoirs, Springer-Verlag Berlin Heidelberg, 2017.

[36] Z. Bassiouni, Theory, Measurement, and Interpretation of Well Logs, Henry L. Doherty Memorial Fund of AIME, Society of Petroleum Engineers, 1994.

[37] M.M.I. Abaza, Streaming Current and Streaming Potential Induced by Water Flow Through Porous Media, Ph.D. thesis, Utah State University, 1996.

[38] K.S. Birdi, Handbook of Surface and Colloid Chemistry, Third Edition, CRC Press, 2008.

[39] J. Wang, H. Hu, W. Guan, H. Li, Electrokinetic experimental study on saturated rock samples: zeta potential and surface conductance, Geophysical Journal International 201 (2015) 869-877.

[40] D.R. Lide, CRC Handbook of Chemistry and Physics, CRC Press, 2004.

[41] P.N. Sen, P.A. Goode, Influence of temperature on electrical conductivity on shaly sands, Geophysics 57 (1992) 89-96.

[42] E. Walker, P.W.J. Glover, J. Ruel, A transient method for measuring the DC streaming potential coefficient of porous and fractured rocks, Journal of Geophysical Research. Solid Earth 119 (2014) 957-970.

[43] P. Glover, G. Schubert (Ed.), Treatise on Geophysics (Second Edition), Elsevier, Oxford, second edition edition, 2015, pp. $89-137$.

[44] A. Revil, P.W.J. Glover, Nature of surface electrical conductivity in natural sands, sandstones, and clays, Geophysical Research Letters 25 (1998) 691-694.

[45] L. Jouniaux, J. Pozzi, Permeability dependence of streaming potential in rocks for various fluid conductivities, Geophysical Research Letters 22 (1995) 485-488.

[46] K. Bjorlykke, Petroleum Geoscience From Sedimentary Environments to Rock Physics, Springer, Berlin, Heidelberg, 2010.

[47] D.L. Johnson, J. Koplik, L. M. Schwartz, New Pore-Size Parameter Characterizing Transport in Porous Media, Phys. Rev. Lett. 57 (1986) 2564-2567.

[48] A. Boleve, A. Crespy, A. Revil, F. Janod, J. L. Mattiuzzo, Streaming potentials of granular media: Influence of the Dukhin and Reynolds numbers, Journal of Geophysical Research B08204 (2007).

[49] P. Sen, C. Scala, M.H. Cohen, A self-similar model for sedimentary rocks with application to the dielectric constant of fused glass beads, Journal of the Soil Mechanics and foundations Division 46 (1981) 781-795. 